\title{
CHARGE RESOLUTION OF PLASTIC TRACK DETECTORS USED TO IDENTIFY RELATIVISTIC NUCLEI
}

\author{
M.H. SALAMON *, P.B. PRICE *, M. TINCKNELL ' and Shi-Lun GUO *** \\ Department of Physics, University of California, Berkeley, California 94720, USA \\ G. TARLÉ \\ Department of Physics, Randall Laboratory, University of Michigan, Ann Arbor, Michigan 48109, USA
}

Received 11 September 1984

\begin{abstract}
We present measurements of charge resolution in the plastic track detectors CR-39(DOP) and Tuffak polycarbonate over the region $10 \leq Z / \beta \leq 105$, determined from plastic stacks exposed to projectile fragments of $1.29 \mathrm{GeV} / \mathrm{u}{ }^{139} \mathrm{La}, 1.45 \mathrm{GeV} / \mathrm{u}{ }^{84} \mathrm{Kr}$, and $1.70 \mathrm{GeV} / \mathrm{u}^{56} \mathrm{Fe}$ produced by nuclear interactions within the stacks, and to $0.96 \mathrm{GeV} / \mathrm{u}^{238} \mathrm{U}$ and $1.0 \mathrm{GeV} / \mathrm{u}{ }^{197} \mathrm{Au}$ ions. The charge resolution obtained is shown to be comparable to the irreducible limit set by fluctuations in energy loss and is consistent with that expected of a track-formation model based on the effects of both $\mathrm{K}$-shell ionization and restricted energy loss.
\end{abstract}

\section{Introduction}

In conceiving new experiments involving charge identification, one needs to know the factors that limit detector resolution. One fundamental limitation that cannot be overcome is that due to Poisson fluctuations in the collision frequency between the charged projectile and electrons within the detector medium, which cause fluctuations in the total energy transferred from the projectile to the detector. Because of the rapid fall-off of the differential spectrum of single-collision energy transfer with electron ( $\delta$-ray) energy, the fluctuations are greater for the large than for the small energy transfers. As reviewed below, dielectric track detectors, which are sensitive mainly to the very low-energy transfers, should ultimately have greater charge-resolving capabilities than ionization and semiconductor detectors, which are sensitive to all classes of energy transfers, and these in turn should be better than nuclear emulsions and organic scintillators, which are primarily sensitive to high-energy transfers ( $\delta$-rays).

Tarlé et al. [1] were the first to demonstrate that a dielectric track detector, CR-39 doped with 1\% dioctylphthalate (denoted CR-39 (DOP)), has a charge reso-

* Also Space Sciences Laboratory, University of California, Berkeley, CA 94720, USA.

+ Also Lawrence Berkeley Laboratory, University of California, Berkeley, CA 94720, USA.

** Present address: Institute of Atomic Energy, Academia Sinica, P.O. Box 275(2), Beijing, China. lution superior to semiconductor detectors of equivalent thickness. They achieved a charge standard deviation $\sigma_{z}=0.24 \mathrm{e} / \sqrt{n}$ for projectile interaction fragments (18 $\leq Z<26$ ) of $1.8 \mathrm{GeV} / \mathrm{u}^{56} \mathrm{Fe}$ ions, a value 2.2 times smaller than that of a $\mathrm{Si}$ detector with equivalent thickness. (Here, $n$ is the number of successive etch pits measured to determine an average etch pit diameter; the relative width of the sum of $\boldsymbol{n}$ independent measurements is $1 / \sqrt{n}$ that of the single measurement distribution. See the top part of fig. 2.) They concluded that the measured charge resolution in CR-39(DOP) was most likely due to chemical nonuniformities within the plastic, or to measurement error, and that fluctuations in energy deposition contributed negligibly to the observed resolution.

In this paper we report charge resolutions measured in two plastic track detectors, CR-39(DOP) and Tuffak [2] polycarbonate, for a variety of charges: in CR39(DOP), $1.0 \mathrm{GeV} / \mathrm{u}{ }^{197} \mathrm{Au}$ ions and fragments with various charges from the nuclear interactions of 1.28 $\mathrm{GeV} / \mathrm{u}{ }^{139} \mathrm{La}, 1.45 \mathrm{GeV} / \mathrm{u}^{84} \mathrm{Kr}$ and $1.7 \mathrm{GeV} / \mathrm{u}{ }^{56} \mathrm{Fe}$ ions; in Tuffak, $0.96 \mathrm{GeV} / \mathrm{u}{ }^{238} \mathrm{U}$ ions and projectile fragments of ${ }^{139} \mathrm{La}$. We find that in both plastics, depending upon the choice of model of track formation, the fluctuations in projectile energy deposition can account for one-half to possibly all of the measured charge resolution, once estimable measurement errors have been subtracted.

In the next section we present experimental procedures and our charge resolution data. The following section reviews the necessary minimum of energy loss 
theory to compare our observations with those predicted by various track formation models.

\section{Response of CR-39(DOP) and Tuffak polycarbonate}

The two plastic track detectors used were $700 \mu \mathrm{m}$ thick CR-39 (Allymer Co. monomer) doped with $1 \%$ dioctylphthalate and $10^{-4}$ Naugard-445 anti-oxidant, made to our specifications by American Acrylic, and $250 \mu \mathrm{m}$-thick Tuffak polycarbonate made by Rohm and Haas [2]. We exposed thick stacks of these materials at nearly normal incidence to the relativistic heavy ion beams, mentioned above, provided by Lawrence Berkeley Laboratory's Bevalac, with a density of $-500 \mathrm{~cm}^{-2}$, and etched the CR-39(DOP) sheets 20 days at $40^{\circ} \mathrm{C}$ in $6.25 \mathrm{~N} \mathrm{NaOH}$ solution and the Tuffak sheets 10 days at $40^{\circ} \mathrm{C}$ in $6.25 \mathrm{~N} \mathrm{NaOH}$ solution saturated with polycarbonate etch products.

To obtain a distribution of particle fragments of accurately known $Z / \beta$ we exploited the fact that at relativistic velocities, fragments emitted in a narrow forward cone, within $\sim 1^{\circ}$ of the beam direction, have almost exactly the beam velocity, and are due to a process called projectile fragmentation in which nucleons are stripped off a projectile nucleus with very little momentum transfer to the fragment [3].

In each stack we scanned the central region of the top sheet with a computer-assisted microscope system and recorded the locations and diameters of $\sim 2500$ primary beam particles, selecting only those in a narrow range of diameters so as to eliminate background fragments with different charge or velocity from the beam. Typically 1 to $2 \%$ of the particles impinging on the stack have interacted somewhere upstream and lost charge or velocity.

Next we selected a sheet at a depth $-2 \mathrm{~cm}$ downstream in the stack and measured the diameters of etch pits on the bottom surface of each sheet formed by surviving beam particles and projectile fragments. With the coordinates of the parent particles in the top sheet as an input to the microscope stage control, the tracks of the surviving beam particles appeared at the expected positions (at these energies Coulomb scattering is negligible in plastic detectors) and the tracks of projectile fragments with $Z \geq 10$ usually appeared displaced by less than one field of view. (The threshold for recording an etch pit in CR-39(DOP) is $Z / \beta \sim 10$; for

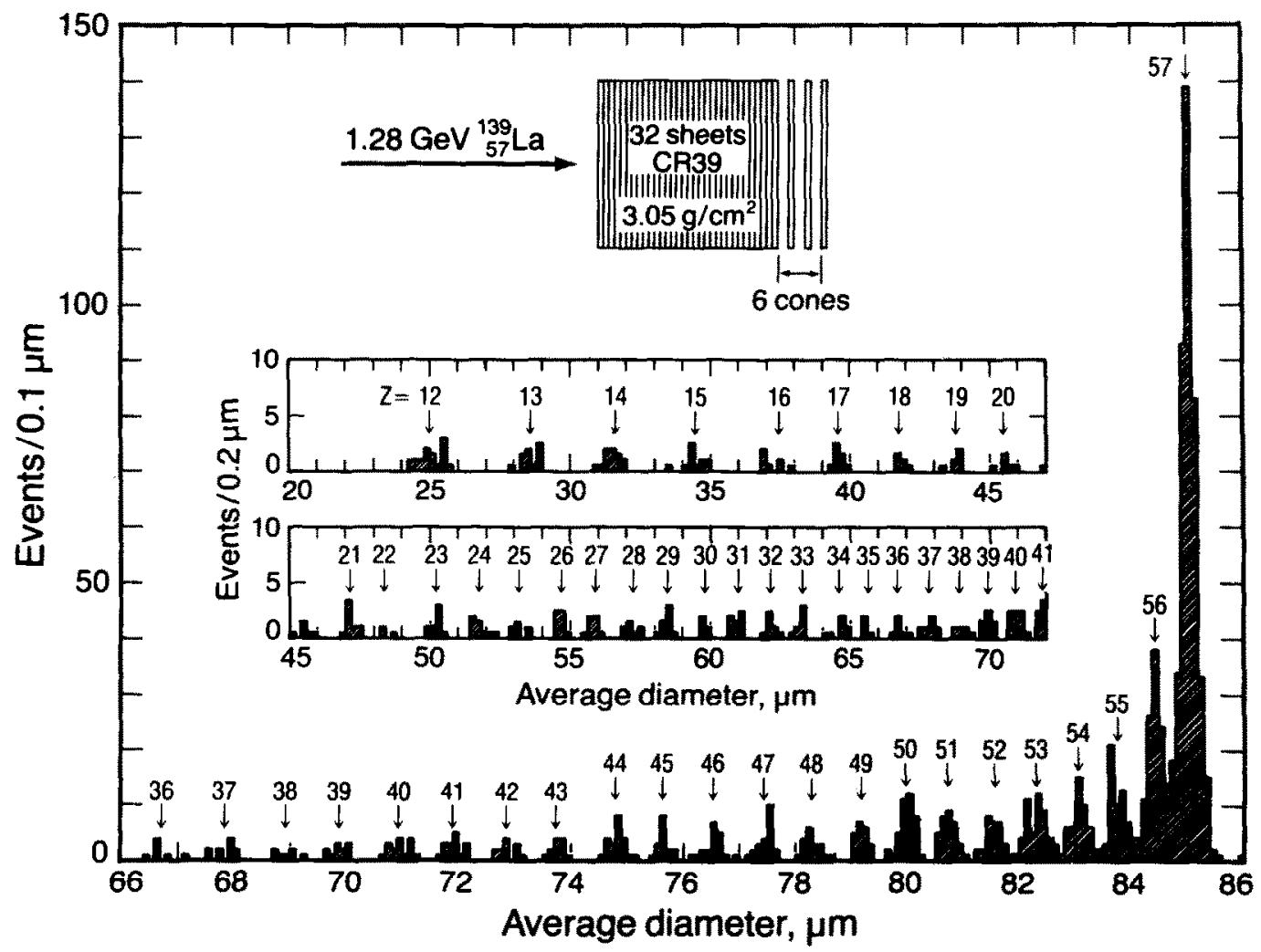

Fig. 1. Diameter histogram of $\mathrm{Kr}$ fragments in CR-39(DOP). These diameters are averages over four consecutive etch pits for each ion. The charge peaks in a single-etch-pit diameter histogram would have full-width-half-maxima twice as large as seen in this figure. Each charge peak is labeled with its charge. 


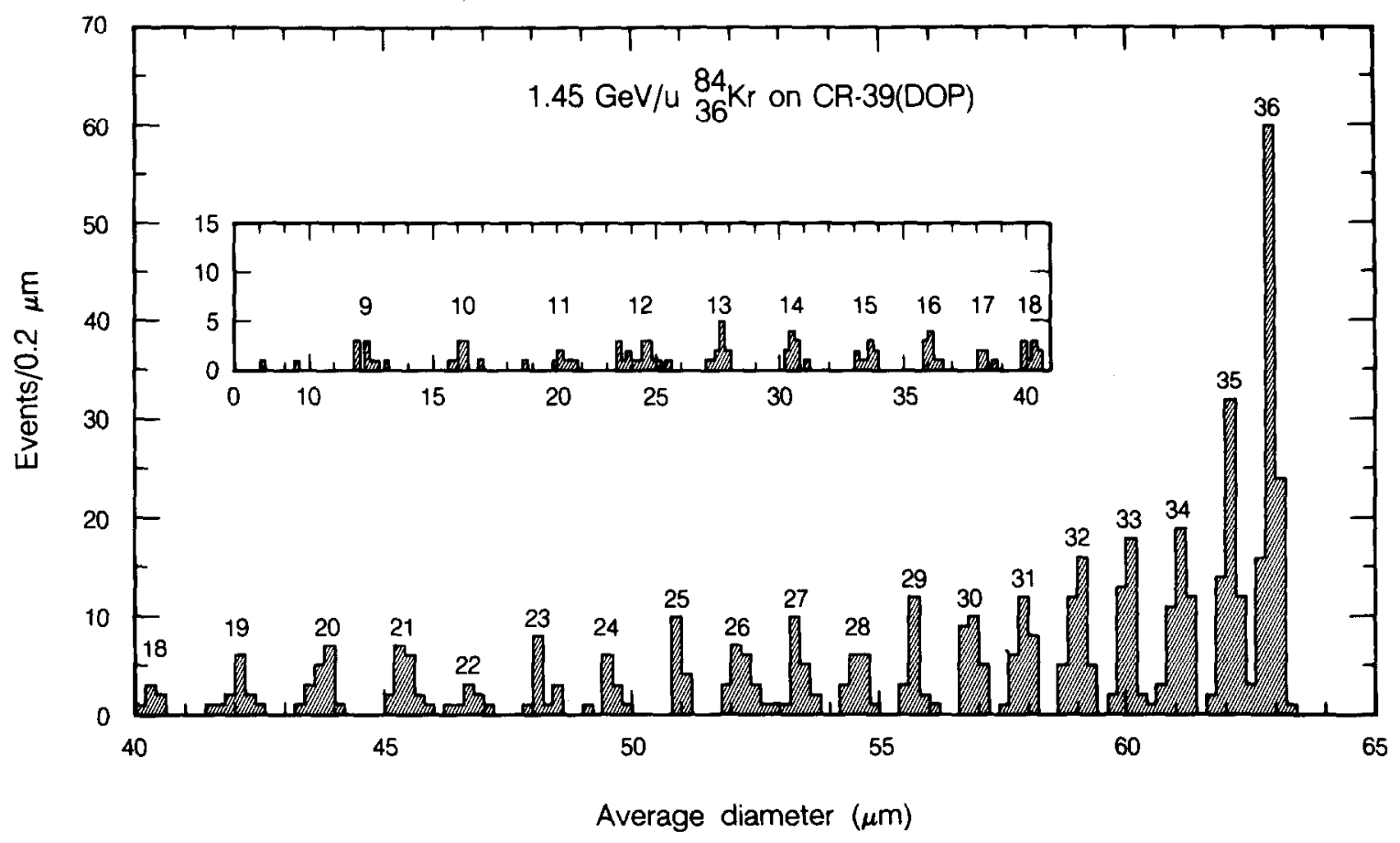

Fig. 2. Diameter histogram of La fragments in CR-39(DOP). These diameters are averages over six consecutive etch-pits for each ion. Each charge peak is labeled with its charge.

Tuffak it is $Z / \beta \sim 55$.) For $\mathrm{Fe}$ and $\mathrm{Kr}$ fragmentation, a negligible fraction of interactions led to two or more projectile fragments with $Z / \beta \geq 10$; for La two projectile fragments with $Z / \beta \geq 10$ were produced in $\sim 1 \%$ of the interactions. In this work we included only the heavier of the two fragments in the charge histograms. Using the track coordinates from the sheet at a depth of $\sim 2 \mathrm{~cm}$ as an input, we located and measured the diameters of the etch pits on the top and bottom surfaces of one or more additional sheets. To reduce measurement labor, we eliminated about two-thirds of the noninteracting particles with a diameter cut before making measurements in the additional sheets. After filtering out the few events in which the etch pit diameter decreased abruptly due to a second fragmentation ( $\sim 10^{-2}$ probability per sheet thickness), we computed the distribution of average etch pit diameters.

Figs. 1 to 3 show the resulting histograms of fouretch-pit $(n=4)$ averages of $\mathrm{Kr}$ fragments in CR39(DOP), of six-etch-pit averages of La fragments in CR-39(DOP), and of four-etch-pit averages of La fragments in Tuffak. In each case the clear separation of individual charge peaks leaves no doubt as to the identity of the fragments.

Fig. 4 shows the measured diameter values as a function of $Z / \beta$ in CR-39(DOP); these diameter measurements were used to calculate the reduced etch rates $s\left(s \equiv v_{\mathrm{T}} / v_{\mathrm{G}}\right.$, where $v_{\mathrm{T}}$ and $v_{\mathrm{G}}$ are the track etch rate and bulk etch rate in the plastic) according to the geometry shown in fig. 5 . The reduced etch rate can be parameterized by one of a number of quantities such as restricted energy loss or $Z / \beta$, over a limited dynamic range; although none of these parameterizations is firmly

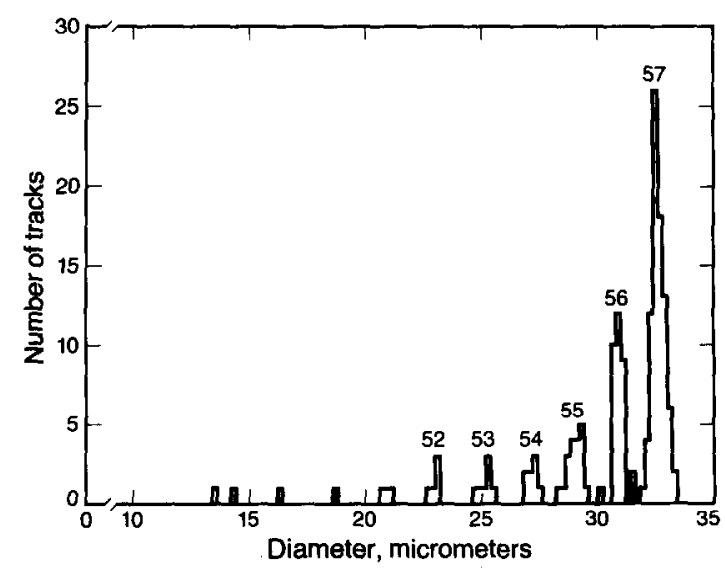

Fig. 3. Diameter histogram of La fragments in Tuffak polycarbonate, with the diameters being averages over four consecutive etch-pits for each ion. In this and the other histograms, ion fragments which interacted a second time within the sheets being measured were filtered out $\left(\sim 10^{-2}\right.$ probability per sheet thickness). 


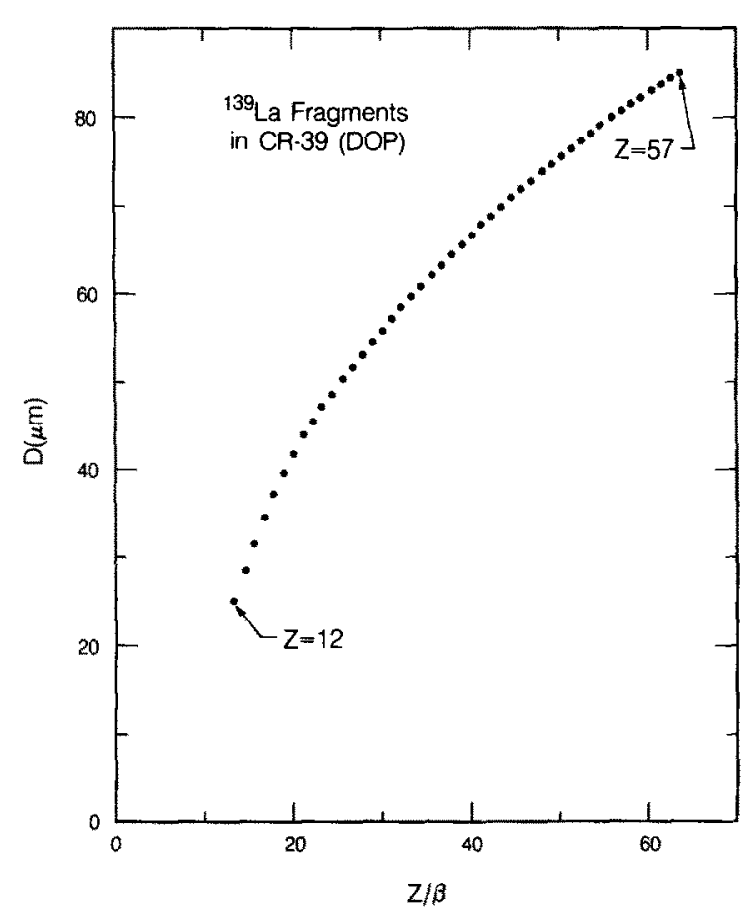

Fig. 4. Measured diameter values $D$ of $\mathrm{La}$ and its fragments as a function of $Z / \beta$ in CR-39(DOP). The function $D(Z / \beta)$ can be trivially converted to $D(Z)$ since the fragment velocities are well known.

theoretically justified [4], for simplicity's sake we choose $s$ versus $Z / \beta$, as displayed in fig. 6 for CR-39(DOP) and Tuffak (data from slowing uranium ions [5] are included in the Tuffak figure). We have found the diameter measurement method to be effective for $(s-1)$ as low as 0.1 to as high as $\sim 12$, corresponding to $12 \leq Z / \beta \leq 80$ and $55 \leq Z / \beta \leq 110$ in $C R-39$ (DOP) and Tuffak, respectively. Above $s \sim 12$ the diameter method's sensitivity to $Z / \beta$ is very low; at this point the more laborious measurements of track length are required for adequate charge resolution, where $\sigma_{z}<1 \mathrm{e}$ has been achieved for slowing uranium ions for $10 \leq Z \leq 60$ [5].

From our data it is clear that a combination of CR-39(DOP) and Tuffak plastic detectors can provide excellent charge resolution over the combined range $12 \leq Z / \beta \leq 110$ using diameter measurements. From the diameter histograms the charge standard deviations $\sigma_{z}$ were computed for each charge peak, $\sigma_{z}=$ $\sigma_{D} /(\partial D / \partial Z)$, where $\sigma_{D}$ is the measured standard deviation of a histogram charge peak and $\partial D / \partial Z$ is the slope of $D(Z)$. No corrections were made for any velocity broadening of the peaks caused by the variability in the location ( 0 to $2 \mathrm{~cm}$ into the stack) of the nuclear interaction producing the fragment, as these were calculated to be negligible.

Figs. 7 and 8 show the distributions of values of $\sigma_{z}$

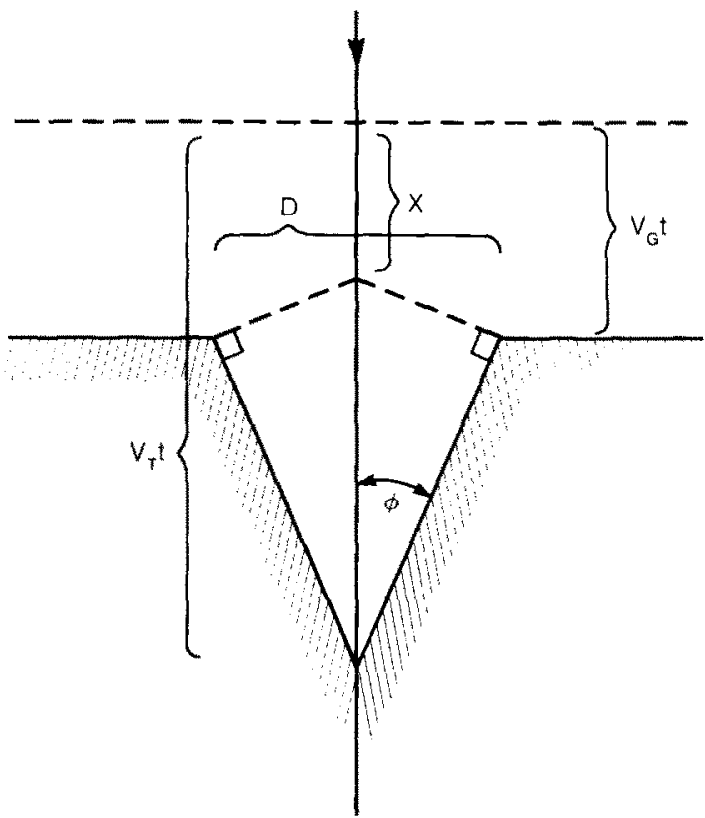

Fig. 5. Geometry of normal-incidence etch-pit. The dashed line is the pre-etch surface of the plastic sheet. During etching, over a time period $t$, the surface is eroded at a general etch rate $v_{\mathrm{G}}$, while the latent track (of several tens of $\AA$ radius) is etched at the faster rate $v_{\mathrm{T}}$. The half-angle $\phi$ of the cone produced is then $=\sin ^{-1}\left(v_{\mathrm{G}} / v_{\mathrm{T}}\right)$, and the final etch pit diameter $D=2 v_{\mathrm{G}} t$ $\sqrt{(s-1) /(s+1)}$, where $s \equiv v_{\mathrm{T}} / v_{\mathrm{G}}$, and normal incidence of the ion is assumed. It is important to note that it is the etch rate within the depth $x$ that completely determines $D$; changes in the etch rate below this depth, although affecting overall track geometry, will not affect the value of $D$. Hence the "active volume" of the detector, when based on diameter mcasurements, has the length $x=v_{\mathrm{G}} t(s /(s+1))$ as shown in the figure for normal incidence.

for a single etch pit $(n=1)$ in CR-39(DOP) and in Tuffak, obtained by multiplying the values of $\sigma_{z}$ for the averages of $n$ etch pits by $\sqrt{n}$. Values of $\sigma_{z}$ in CR39 (DOP) for fragments of $1.85 \mathrm{GeV} / \mathrm{u}{ }^{40} \mathrm{Ar}$, previously published elsewhere [6], are included in fig. 7 for completeness; the etching and analysis procedures for this stack were essentially the same as described above. Included in these figures are values of $\sigma_{z}$ for beams of $1.0 \mathrm{GeV} / \mathrm{u}{ }^{197} \mathrm{Au}$ in CR-39(DOP) and for $0.96 \mathrm{GeV} / \mathrm{u}$ ${ }^{238} \mathrm{U}$ in Tuffak. The error bars shown in figs. 7 and 8 are due to the counting statistics associated with each charge peak in the fragment distribution.

A weighted, least squares fit of all the CR-39(DOP) charge standard deviation data to a second-order polynomial in $Z / \beta$ yields, in the region $10 \leq Z / \beta \leq 90$,

$$
\begin{aligned}
\sigma_{z}= & \frac{1}{\sqrt{n}}\left[0.194+1.94 \times 10^{-3}\left(\frac{Z}{\beta}\right)\right. \\
& \left.+4.35 \times 10^{-5}\left(\frac{Z}{\beta}\right)^{2}\right] \mathrm{e} .
\end{aligned}
$$




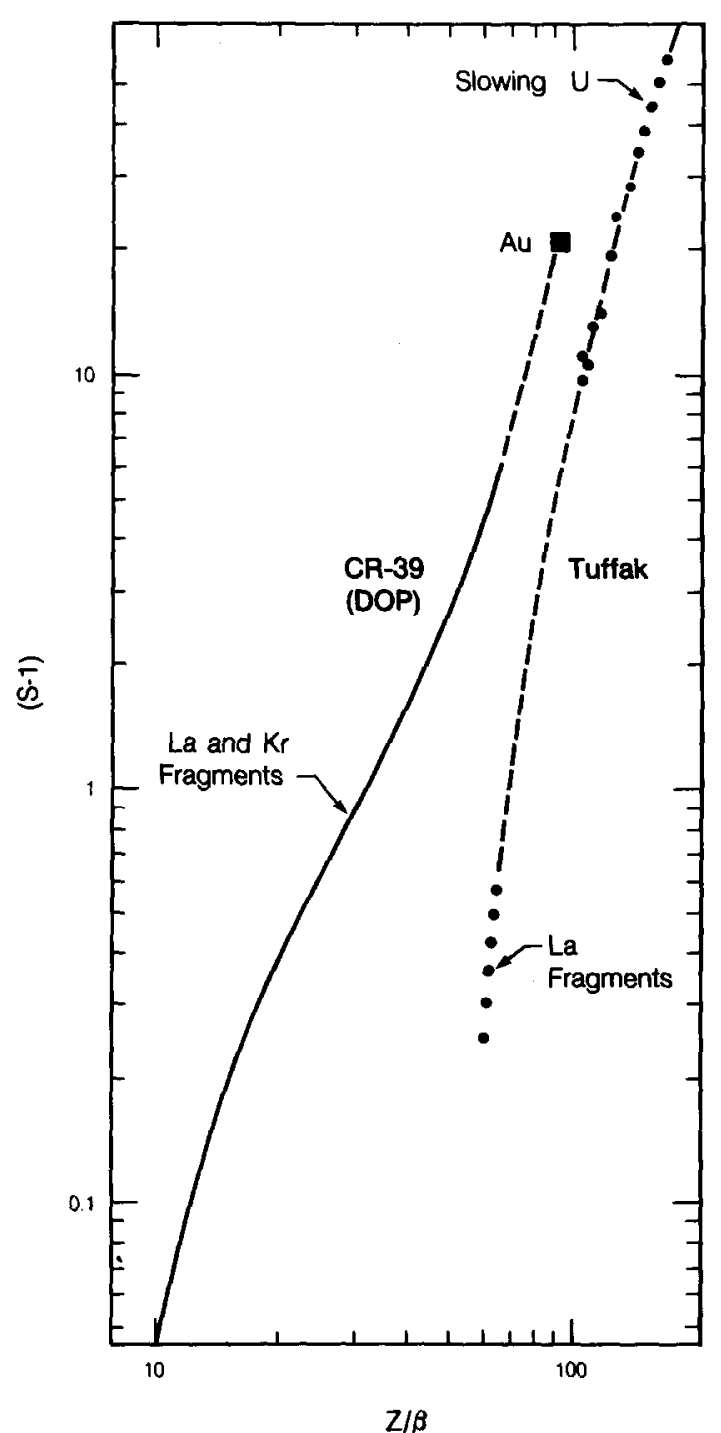

Fig. 6. Reduced etch rate $s \equiv v_{\mathrm{T}} / v_{\mathrm{G}}$ versus $Z / \beta$ for various relativistic heavy ions in both CR-39(DOP) and Tuffak polycarbonate plastics. The dashed lines are regions of interpolation between data. The data on which the curves are based are sufficiently precise to rule out simple power-law fits.

Again, $n$ is the number of successive etch pits used to obtain an average diameter. An assumption in predicting a narrowing of $\sigma_{z}$ by $1 / \sqrt{n}$ is that these measurements are statistically independent, which is not correct for the higher charges where significant electron attachment occurs even at relativistic energies. This problem and its solution have been discussed in detail elsewhere [7]. It should be emphasized that the specific values of $\sigma_{z}$ obtained here are dependent upon the bulk thickness of plastic removed during etching (see below); $51 \mu \mathrm{m}$ was removed from each surface of the CR-39(DOP) sheets, while $35 \mu \mathrm{m}$ was removed from each Tuffak sheet surface.

For Tuffak, the number of measurements of $\sigma_{z}$ is too small to permit us to derive a meaningful empirical relation between $\sigma_{z}$ and $Z / \beta$ over the interval $60 \leq Z \leq$ 105; a simple, weighted least squares linear fit, however, gives

$\sigma_{z}=\left[-0.618+1.51 \times 10^{-2}\left(\frac{Z}{\beta}\right)\right] \mathrm{e} \quad$ for $n=1$.

We note that in the region $Z / \beta-60$, Tuffak has a significantly better charge resolution than does $C R$ 39 (DOP), and at values of $Z / \beta$ up to at least $\sim 105$ one can obtain a single-etch-pit value of $\sigma_{z}<1$ e with Tuffak using the diameter method.

\section{Contribution of fluctuations in energy loss to observed charge variances}

At present there does not exist a successful theory of track formation in plastics. A number of semi-phenomenological models exist $[8,9]$, but their predictive power is limited at best, in part because they do not adequately treat the complex chemical processes that must certainly take place in latent track formation and during etching. Most models assume track damage in plastic to be some function of a quantity related to the projectile energy loss: this quantity can be either the ion's total energy loss, a restricted energy loss (defined below), the ion's primary ionization rate, or the dose (total energy density) at a specific distance from the projectile path $[8,9]$. The specific choice reflects assumptions about the physical mechanisms involved in track formation. No one parameterization seems to adequately fit all data, however; in polycarbonate plastics, for example, the etch rate is better represented as a function of $Z / \beta$ than restricted energy loss [4], while the opposite appears to be the case in CR-39(DOP). (For the data presented here, both parameterizations are essentially equivalent because our heavy ion velocities are roughly constant at $\beta \sim 0.9$.)

In contrast to plastics, the state of track formation theory for minerals, which are physically simpler systems, is more advanced [10]. Small-angle X-ray scattering by latent tracks in silicates by Dartyge et al. [11] has shown that latent tracks are composed of "point" defects and "extended" defects. The observed chemical etch rate is a function of the linear densities of these defects, with the observed etch rate being dominated by the presence of extended defects. It is observed that the linear density of point defect closely follows projectile energy loss along the track, while the density of extended defects does not. Tombrello et al. [12-14] suggest that extended defects are generated by atomic $\mathrm{K}$-shell excitations in the heavier elements of the mineral. 


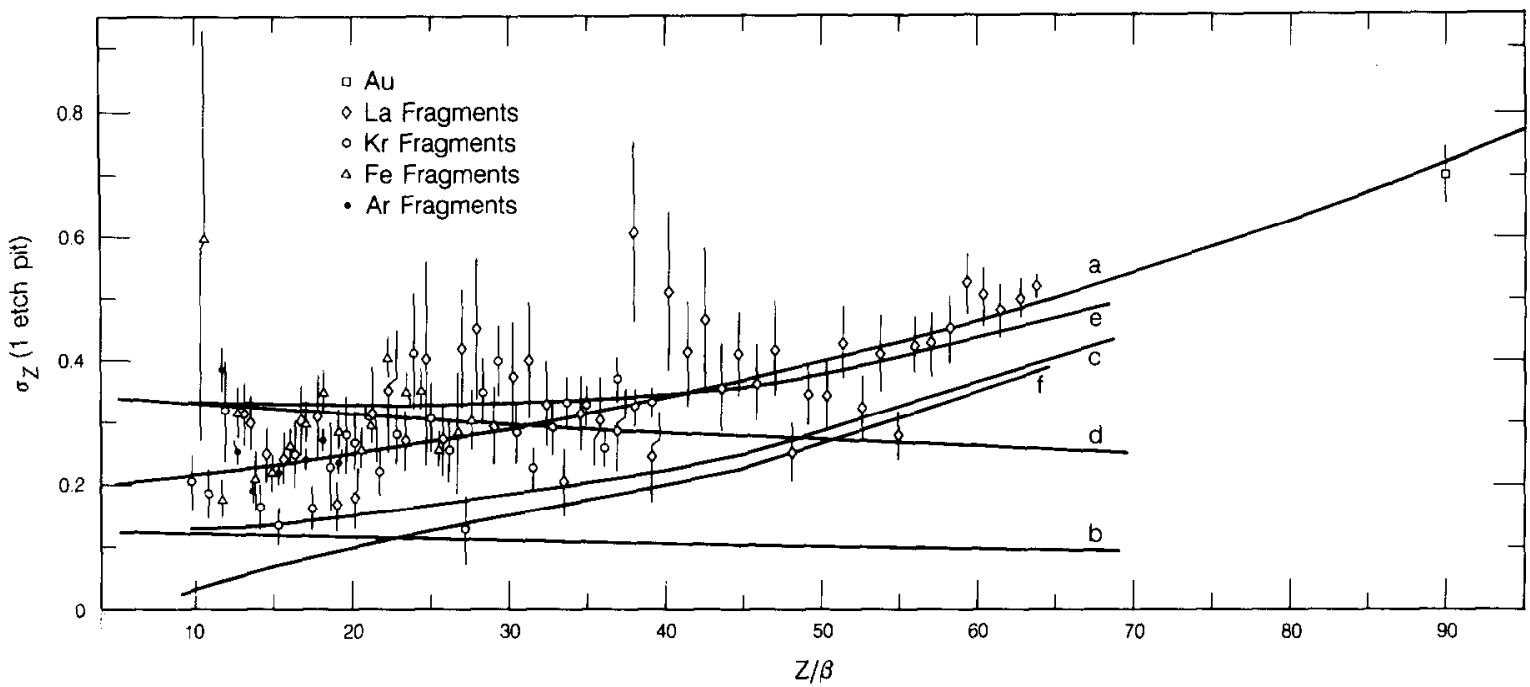

Fig. 7. Measured charge resolution $\sigma_{z}$ for a single etch pit $(n=1)$ as a function of $Z / \beta$ in CR-39(DOP), obtained from the charge peak widths of the fragment distributions of La (fig. 2), $\mathrm{Kr}$ (fig. 1), $\mathrm{Fe}$, and Ar ions that have interacted with the detector stack. The labeled curves are a) weighted least squares quadratic fit to the data, b) calculated charge resolution $\sigma_{z}^{\prime}$ based on fluctuations in a restricted energy loss model $\left(\omega_{0}=0.20 \mathrm{keV}\right)$, c) curve b with calculated measurement error $\sigma_{z}$ (measurement) added in quadrature, d) calculated $\sigma_{z}^{\prime}$ based on fluctuations in a K-shell excitation model, e) curve $d$ with calculated $\sigma_{z}$ (measurement) added in quadrature, $f$ ) the calculated error $\sigma_{z}$ (measurement) alone.

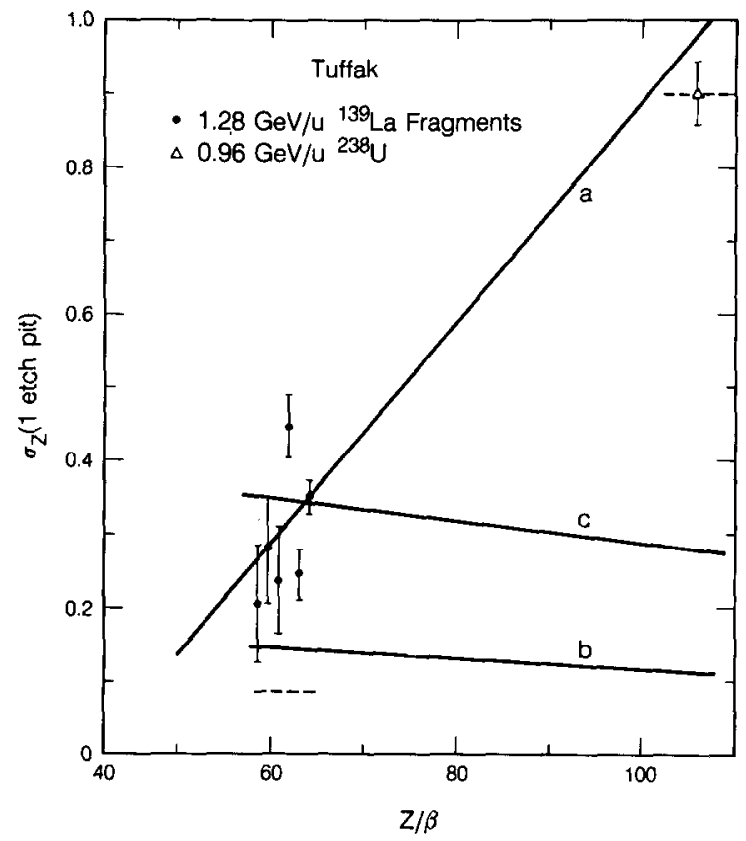

Fig. 8. Measured charge resolution $\sigma_{z}$ for a single etch pit $(n=1)$ as a function of $Z / \beta$ in Tuffak polycarbonate, obtained from the diameter histogram of La projectile fragments (fig. 3), and from the width of the diameter distribution of $0.96 \mathrm{GeV} / \mathrm{u}$ ${ }^{238} \mathrm{U}$ ions. The labeled curves are a) weighted least squares linear fit to the data, b) calculated charge resolution $\sigma_{z}^{\prime}$ based on fluctuations in a restricted energy loss model $\left(\omega_{0}=0.35\right.$ $\mathrm{keV}$ ), c) calculated $\sigma_{z}^{\prime}$ based on fluctuations in a $\mathrm{K}$-shell excita-
The Auger decay of an inner shell vacancy produces a multiply-charged ion whose Coulomb interaction with the surrounding ionized medium causes the atomic motion that produces the observed defect [13]. This model is quite successful in explaining the morphology and frequency of extended defects [14] and gives good agreement with ion sputtering data as well [12].

It is conceivable that we can apply a similar model to plastics, in which most of the etchable track damage is due to the interaction of Auger-decayed K-shell vacancies in oxygen and carbon atoms with the roughly uniform density of outer-shell ionized ions. For reasons given below, the outer-shell ionization density that contributes to track formation is probably most closely related to restricted energy loss, while the $\mathrm{K}$-shell excitation rate can be estimated via collision theory. Because of the complex chemistry of organic polymers, however, it is large step from postulating initial damage mechanisms to predicting observed etch rates. Nevertheless, there is an indirect method of testing models of damage that bypasses the complex chemistry, and that is by examining fluctuations of etch rate.

tion model. Our automated microscope system measures diameters with a stochastic reading error of $\sim 0.15 \mu \mathrm{m}$; for our Tuffak plastic this is the dominant error contributing to the observed charge resolution. Dashed line segments give $\sigma_{z}$ (measurement) at the $\mathrm{La}$ and $\mathrm{U}$ points. 
Although the reduced etch-rate $s$ may be a formidable function of energy loss and other quantities, the energy loss itself (or restricted energy loss, $\mathrm{K}$-shell ionization rates, etc.) is proportional to $Z^{2}$ to first order in scattering theory, where $Z$ is the projectile charge. Therefore, fluctuations in energy loss are reflected in the observed charge resolution by the simple relation $\sigma_{E} / \Delta E=2 \sigma_{z} / Z$, where $\sigma_{E}$ is the standard deviation of the energy loss distribution whose mean is $\Delta E$. Measurements of charge resolution then can place constraints on $\sigma_{E}$, which is model-dependent. Implicit in this equation is the removal of other factors contributing to a finite charge resolution $\sigma_{z}$, such as measurement error, inhomogeneities in composition, etc.

We therefore compare our experimental charge resolution data to those predicted by models which assume either a) that the observed etch rate $s$ is a (complicated) function solely of restricted energy loss, or b) that $s$ is a function solely of the number of $\mathrm{K}$-shell excitations. The observed $\sigma_{z}(Z)$ is found to be consistent with some combination of these two processes, as expected from track models in minerals.

\subsection{Restricted energy loss models}

Transmission electron microscope observations and electrical measurements of conductivity through etched pores [8] have shown that the region of intense radiation damage along a track has a lateral extent of only a few tens of angströms, and measurements of the rate of diameter growth during etching around a particle's trajectory show that the region of enhanced chemical reactivity, the "active volume", has a similar lateral extent ]8]. Since electrons suffering large energy transfers deposit most of the energy outside the active volume, it is plausible that the active volume about the particle trajectory is formed primarily by electrons whose transfer energy $\omega$ is below a cutoff value, $\omega_{0}$. (In a Tombrello-type model, these electrons would produce the outer-shell ionization density.) For models of track formation which make this assumption, $\omega_{0}$ is typically $<1 \mathrm{keV}$. Assuming that the reduced etch rate $s$ is an empirical function solely of the restricted energy loss $\mathrm{d} E / \mathrm{d} x\left(<\omega_{0}\right)$ (the total energy loss per unit distance to electrons with energy transfers $<\omega_{0}$ ), Benton and Henke [15] obtained a best fit value of $0.35 \mathrm{keV}$ for heavy ion tracks in Lexan polycarbonate (a polymer identical in composition to Tuffak), and Fowler et al. [16] obtained $\omega_{0} \approx 0.20 \mathrm{keV}$ for CR-39.

We now calculate the effect of energy loss fluctuations in those models of track formation which assume that the reduced etch rate $s$ is solely a function of the restricted energy loss $\mathrm{d} E / \mathrm{d} x\left(<\omega_{0}\right)$. In this case, only the fluctuations in total energy loss to electrons with energy transfer less than $\omega_{0}$ contribute to the measured charge variance. When $\omega_{0}=\infty$, the variance $\sigma_{E}^{2}$ in total energy loss is given by the traditional Bohr formula,

$\sigma_{E}^{2}=x \int_{\omega_{\min }}^{\omega_{\max }} \frac{\mathrm{d} n_{\mathrm{e}}}{\mathrm{d} x \mathrm{~d} \omega} \omega^{2} \mathrm{~d} \omega=\xi \omega_{\max }\left[1-\frac{\omega_{\max }}{4 m c^{2} \gamma^{2}}\right]$,

where

$\frac{\mathrm{d} n_{e}}{\mathrm{~d} x \mathrm{~d} \omega}=\frac{2 \pi N Z^{2} e^{4}}{m c^{2} \beta^{2} \omega^{2}}\left(1-\frac{\omega}{2 m c^{2} \gamma^{2}}\right)$

is the differential spectrum of energy transfer $\omega$ to electrons in collision with the projectile ion of charge $Z$ and velocity $\beta$ (in units of speed of light, $c$ ), $N$ is the electron density in the medium, $x$ is the projectile pathlength over which the energy loss $\mathrm{d} E / \mathrm{d} x$ is integrated, $\omega_{\max }$ is the maximum $\delta$-ray energy (which is the kinematic limit $2 \mathrm{mc}^{2} \beta^{2} \gamma^{2}$ for non-restricted energy loss variance), $\omega_{\min }$ is some minimum energy transfer $\ll$ $\omega_{\max }$, which contributes negligibly to the standard Bohr integral, and $\xi \equiv 2 \pi N Z^{2} e^{4} x / m c^{2} \beta^{2}$.

Fano [17], however, has shown that when the statistics associated with low energy transfers are properly taken into account, one obtains

$\sigma_{E}^{2}($ total $)=\xi\left[2 m c^{2} \beta^{2} \gamma^{2}\left(1-\beta^{2} / 2\right)+\frac{8}{3}\langle K\rangle \ln \frac{2 m c^{2} \beta^{2}}{I_{1}}\right]$

where $\langle K\rangle$ is the mean energy of an atomic electron in the ground state of the absorbing atom, and $I_{1}$ is a weighted mean ionization energy (not the usual mean ionization energy $I_{\text {adj }}$ found in stopping power formulae). Ahlen [4] has estimated these to be $\langle K\rangle=0.123$ $\mathrm{keV}$ and $I_{1}=0.323 \mathrm{keV}$ in plastics, to an accuracy of $\pm 50 \%$. Since in plastics there is no sensitivity to fluctuations for $\omega>\omega_{0}$, the contribution to $\sigma_{E}^{2}$ for $\omega_{0}<$ $\omega \leq 2 m c^{2} \beta^{2} \gamma^{2}$ (the kinematic $\delta$-ray limit) must be subtracted out [4], viz.,

$\sigma_{E}^{2}\left(<\omega_{0}\right)=\sigma_{E}^{2}($ total $)-x \int_{\omega_{0}}^{2 m c^{2} \beta^{2} \gamma^{2}} \frac{\mathrm{d} n_{\mathrm{e}}}{\mathrm{d} x \mathrm{~d} \omega} \omega^{2} \mathrm{~d} \omega$,

which yields

$\sigma_{E}^{2}\left(<\omega_{0}\right)=\xi\left[\omega_{0}+\frac{8}{3}\langle K\rangle \ln \frac{2 m c^{2} \beta^{2}}{I_{1}}-\frac{\omega_{0}^{2}}{4 m c^{2} \gamma^{2}}\right]$.

This is the variance for the restricted energy loss $\Delta E(<$ $\left.\omega_{0}\right) \approx x \mathrm{~d} E / \mathrm{d} x\left(<\omega_{0}\right)$, where (see Fano [17]),

$\frac{\mathrm{d} E}{\mathrm{~d} x}\left(<\omega_{0}\right)=\frac{\xi}{x}\left[\ln \frac{2 m c^{2} \beta^{2} \gamma^{2} \omega_{0}}{I_{\mathrm{adj}}^{2}}-\beta^{2}\right]$,

with $I_{\text {adj }} \approx 0.070 \mathrm{keV}$ for plastics.

The pathlength $x$ in the above formulae is the thickness of plastic along the projectile trajectory that is causally related to the magnitude of the diameter of the etch pit. Examination of fig. 5 shows that fluctuations in the final diameter value can be affected only by fluctuations in etching along the active depth $x=v_{\mathrm{G}} t(s / s+1)$ for particles at normal incidence, where $t$ is the total etching time, and $s$ is the reduced etch rate. 
Ignoring the weak dependence of $x$ on $Z / \beta, \Delta E(<$ $\left.\omega_{0}\right)$ is proportional to $Z^{2}$, so that $\sigma_{z}^{\prime}=(Z / 2) \cdot\left(\sigma_{E}(<\right.$ $\left.\left.\omega_{0}\right) / E\left(<\omega_{0}\right)\right)$, where the prime distinguishes the theoretically calculated charge standard deviation from the experimental value $\sigma_{z}$. Using the values $\omega_{0}=0.20 \mathrm{keV}$ [16] in CR-39(DOP) and $\omega_{0}=0.35 \mathrm{keV}$ [15] in Tuffak, $\sigma_{z}^{\prime}$ is calculated and compared to data in figs. 7 (curve b) and 8.

Concentrating on the more abundant CR-39(DOP) data, we note two features of this fit: first, the $\sigma_{z}$ data arc at least twice as large as $\sigma_{z}^{\prime}$, and second, $\sigma_{z}^{\prime}$ decreases slowly with $Z$, while $o_{z}$ increases. By treating $\omega_{0}$ as a free parameter, one obtains a fit to the lower charge data only for $\omega_{0}=14 \mathrm{keV}$, much larger than is usually accepted in restricted energy loss models of track formation. This still does not account for the rise in $\sigma_{z}$ with $Z$, which is most probably due to measurement error. (Nonuniformities in plastic composition may also contribute, although large-scale inhomogeneities have been measured and are insignificant.) If the particle incidence angle is a few degrees away from normal, then the etch pit circles are in fact ellipses of finite eccentricity, so that etch pit diameters now vary depending upon whether the major or minor axis is being measured. In fact, during our measurements we did not distinguish between these two since we assumed normal incidence; however, re-examination of the CR-39(DOP) sheets showed a $3^{\circ}$ off-normal incidence. In addition, we estimate a constant diameter measurement error of 0.15 $\mu \mathrm{m}$ using our automated microscope system which would add in quadrature to the above error. Fig. 7 , curve $f$, shows the calculated $\sigma_{z}$ (measurement) as a function of $Z$ for $0.15 \mu \mathrm{m}$ constant measurement error plus $4^{\circ}$ off-normal incidence with no distinction made between major and minor axis diameters (an extra $1^{\circ}$ is added to account for non-perfect alignment of optic axis with the surfaces of the examined sheets). It is clear that measurement errors have a negligible effect on charge resolution for the lower charges where $\partial D / \partial Z$ (fig. 4) is largest; it is also clear that for the higher charges the observed resolution is dominated by measurement error, so that our physical interpretation will be based on the measured $\sigma_{z}$ for the lower charges only. Fig. 7, curve c, shows $\left[\sigma_{z}^{2}\right.$ (measurement $\left.)+\sigma_{z}^{\prime 2}\left(\omega<\omega_{0}=0.20 \mathrm{keV}\right)\right]^{1 / 2}$ which is the calculated charge resolution due to intrinsic energy loss fluctuations and to estimated measurement errors; it is apparent that the predicted charge resolution is lower than observed, by about a factor of two.

\section{2. $K$-shell excitation model}

We next calculate the expected charge resolution for models in which the track etch rate is solely a function of the number of $\mathrm{K}$-shell ionizations within the active volume. (We note that the Tombrello model is not of this class, since in that model the ionization energy density surrounding the track also plays a rolc. Although the general ionization and excitation density about the Auger-decayed atoms follows a restricted energy loss, it is clear that fluctuations in response will be dominated by the statistics of K-shell excitations because there are fewer of these excitations.) One can quickly estimate the number of $\mathrm{K}$-shell excitations produced by a relativistic projectile along a given pathlength by using the Bethe-Bloch stopping power relation

$\frac{\mathrm{d} E}{\mathrm{~d} x}=\frac{4 \pi N Z^{2} e^{4}}{m c^{2} \beta^{2}}\left[\ln \frac{2 m c^{2} \beta^{2} \gamma^{2}}{I_{\mathrm{adj}}}-\beta^{2}\right]$,

where $N \ln I_{\text {adj }} \equiv \sum_{i} N_{i} \ln E_{i}$, and $N_{i}=$ number density of electrons at energy level $E_{i}$. Restricting the sum to $\mathrm{K}$-shell electrons of oxygen $(\mathrm{O})$ and carbon $(\mathrm{C})$, one obtains

$$
\begin{aligned}
\left(\frac{\mathrm{d} E}{\mathrm{~d} x}\right)_{\mathrm{K}}= & \frac{4 \pi Z^{2} e^{4}}{m c^{2} \beta^{2}}\left[N_{\mathrm{K}, \mathrm{o}} \ln \frac{2 m c^{2} \beta^{2} \gamma^{2}}{E_{\mathrm{K}, \mathrm{O}}}\right. \\
& \left.+N_{\mathrm{K}, \mathrm{C}} \ln \frac{2 m c^{2} \beta^{2} \gamma^{2}}{E_{\mathrm{K}, \mathrm{C}}}-\left(N_{\mathrm{K}, \mathrm{O}}+N_{\mathrm{K}, \mathrm{C}}\right) \beta^{2}\right] .
\end{aligned}
$$

The average number of $\mathrm{K}$-shell excitations along the active depth $x$ is then roughly given by

$$
\begin{aligned}
n_{\mathrm{K}}= & \frac{4 \pi Z^{2} e^{4} x}{m c^{2} \beta^{2}}\left[\frac{N_{\mathrm{K}, \mathrm{O}}}{E_{\mathrm{K}, \mathrm{O}}} \ln \frac{2 m c^{2} \beta^{2} \gamma^{2}}{E_{\mathrm{K}, \mathrm{O}}}\right. \\
& \left.+\frac{N_{\mathrm{K}, \mathrm{C}}}{E_{\mathrm{K}, \mathrm{C}}} \ln \frac{2 m c^{2} \beta^{2} \gamma^{2}}{E_{\mathrm{K}, \mathrm{C}}}-\left(\frac{N_{\mathrm{K}, \mathrm{O}}}{E_{\mathrm{K}, \mathrm{O}}}+\frac{N_{\mathrm{K}, \mathrm{C}}}{E_{\mathrm{K}, \mathrm{C}}}\right) \beta^{2}\right] .
\end{aligned}
$$

Since the adiabatic impact parameters [18] for carbon and oxygen K-shell ionization are of the order of $10 \AA$, certainly within the radius of track damage in plastics as determined by electrical measurements [8], all the K-shell excitations contribute to the formation of track damage. We have also calculated $n_{\mathrm{K}}$ directly using published cross-sections of $\mathrm{K}$-vacancy production by highly relativistic protons [19]; those calculations yield the same numerical results.

Making the simplistic assumption that the measured etch pit diameters are solely a function of $n_{\mathrm{K}}$, it follows that $\sigma_{z}^{\prime}=Z / 2 \sqrt{n_{K}}$ (from Poisson statistics), which is plotted in fig. 7 (curve d). Combining $\sigma_{z}^{\prime}$ in quadrature with the measurement error $\sigma_{z}$ (measurement) discussed above produces curve $e$ of fig. 7 , which is somewhat higher than the data. Applying this model to the Tuffak data, we see in fig. 8 that again the restricted energy loss model's prediction of $\sigma_{z}$ is too low, while the K-shell excitation model's $\sigma_{z}^{\prime}$, combined in quadrature with a constant measurement error of $0.15 \mu \mathrm{m}$ (the incidence angle in the Tuffak stack was $\sim 0^{\circ}$ ) is again somewhat higher than the measured charge resolution data. 


\subsection{Conclusion}

The fact that our observed charge resolution $\sigma_{z}(Z)$ in both Tuffak polycarbonate and in CR-39(DOP) lies between the values $\sigma_{z}^{\prime}$ calculated by the K-shell excitation model and the restricted energy loss model is suggestive of the possibility that the energy-loss process responsible for track formation in plastics is a combination of these, such as discussed by Tombrello for minerals. Our data rules out, for example, any models in which tracks are dependent upon total energy loss or solely dependent upon $\mathrm{K}$-shell excitation rates (the calculated charge resolutions $\sigma_{z}^{\prime}$ are too large), and also rules out pure restricted energy loss models that use a reasonable value for the cutoff energy $\omega_{0}$ (the $\sigma_{z}^{\prime}$ are too low). Although we feel that we have carefully considered all likely sources of error, it is possible that we have not accounted for all sources of error in the lower charge region; this would vitiate our conclusion regarding pure restricted energy loss models.

We note parenthetically that there is other evidence that supports a K-shell excitation/restricted energy loss model. A feature not explained by pure restricted energy loss models is the closeness of the $\omega_{0}$ values for CR-39(DOP) and Tuffak, $0.20 \mathrm{keV}$ and $0.35 \mathrm{keV}$, despite the dramatic difference in sensitivity of these plastics. (Significant bulk damage occurs at 2 Mrad in CR-39(DOP) and at $100 \mathrm{Mrad}$ in polycarbonates [20].) If it is the Coulomb interaction between the Auger-decayed ions and the surrounding ionized medium that causes track damage, then there is most likely a cutoff radius beyond which the Auger-decayed ion is unable to cause damage. This cutoff radius, insensitive to plastic type, would then be of the order of the projected range of a delta-ray of energy $\omega_{0}$, so that only the restricted energy loss $\mathrm{d} E / \mathrm{d} x\left(<\omega_{0}\right)$, in concert with $\mathrm{K}$-shell excitations, would ultimately contribute to track damage.

\section{Summary}

We have demonstrated that CR-39(DOP) and Tuffak polycarbonate track detectors in concert can provide excellent charge resolution over the combined range $12 \leqq Z / \beta \leqq 110$ using diameter measurements, with a corresponding charge resolution of $0.22 \leq \sigma_{z} \leq 0.90 \mathrm{e}$ for measurement of a single etch pit. The great advantage to this technique is that measured charge resolution $\sigma_{z}$ diminishes as $1 / \sqrt{n}$, where $n$ successive etch pit diameters are averaged for each ion (see ref. [7] for exceptions, however), so that with additional measurements $\sigma_{z}$ can be made almost arbitrarily low (of course, multiple measurements help only over a stack thickness less than a mean free path for nuclear interactions).
Fluctuations in energy deposition by the projectile ion within the plastic medium produce an irreducible source of dispersion in any measured charge distribution. The charge resolutions we have measured have been compared to those expected from certain classes of track production models. On the basis of the measured charge resolutions alone we are able to eliminate the viability of certain types of models; our data is found to be consistent with a model in which Auger-decayed inner-shell vacancies of $\mathrm{C}$ and $\mathrm{O}$ atoms interact with the surrounding outer-shell-ionized medium to produce defects of enhanced etch rate.

\section{References}

[1] G. Tarlé, S.P. Ahlen and P.B. Price, Nature 293 (1981) 556.

[2] D. O'Sullivan and A. Thompson, Proc. 11th Int. Conf. on Solid State Nuclear Track Detectors (Pergamon, New York, 1982) p. 85.

[3] D.E. Greiner, P.J. Lindstrom, H.H. Heckman, B. Cork and F.S. Bieser, Phys. Rev. Lett. 35 (1975) 152.

[4] S.P. Ahlen, Rev. Mod. Phys. 52 (1980) 121.

[5] Shi-lun Guo, J. Drach, P.B. Price, M.H. Salamon, G. Tarlé, M.L. Tincknell and S.P. Ahlen, Nucl. Tracks (1984), in press.

[6] P.B. Price, M.L. Tincknell, G. Tarlé, S.P. Ahlen, K.A. Frankel and S. Perlmutter, Phys. Rev. Lett. 50 (1983) 566.

[7] M.H. Salamon, J. Drach, Shi-lun Guo, P.B. Price, G. Tarlé and S.P. Ahlen, Nucl. Instr. and Meth. 224 (1984) 217.

[8] R.L. Fleischer, P.B. Price and R.M. Walker, Nuclear Tracks in Solids (University of California Press, Berkeley, 1975).

[9] E.V. Benton, Rad. Effects 2 (1970) 273.

[10] P. Pellas and C. Perron, Nucl. Instr. and Meth. B1 (1984) 387.

[11] E. Dartyge, J.P. Durand, Y. Langevin and M. Maurette, Phys. Rev. B23 (1981) 5213.

[12] T.A. Tombrello, Nucl. Instr. and Meth. B1 (1984) 23.

[13] T.A. Tombrello, C.R. Wie, N. Itoh and T. Nakayama, Phys. Lett. 100A (1984) 42.

[14] T.A. Tombrello, Nucl. Instr. and Meth., in press.

[15] E.V. Benton and R.P. Henke, University of San Francisco Technical Report No. 19 (1972).

[16] P.H. Fowler, S. Amin, V.M. Clapham and D.L. Henshaw, Proc. 10th Int. Conf. on Solid State Nuclear Track Detectors (Pergamon, New York, 1980), p. 239.

[17] U. Fano, Ann. Rev. Nucl. Sci. 13 (1963) 1

[18] J.D. Jackson, Classical Electrodynamics (Wiley, New York, 1975), p. 618

[19] R. Anholt, S. Nagamiya, J.O. Rasmussen, H. Bowman, J.G. Ioannou-Yannou and E. Rauscher, Phys. Rev. A14 (1976) 2103.

[20] D. O'Sullivan, P.B. Price, K. Kinoshita and C.G. Willson, J. Electrochem. Soc. 129 (1982) 811. 